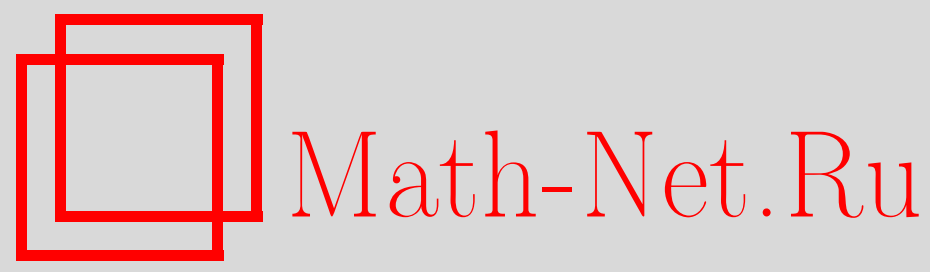

В. В. Белокуров, Ю. П. Соловьев, Е. Т. Шавгулидзе, Метод суммирования расходящихся рядов с любой точностью, Матем. заметки, 2000, том 68, выпуск 1, 24-35

DOI: https://doi.org/10.4213/mzm916

Использование Общероссийского математического портала Math-Net.Ru подразумевает, что вы прочитали и согласны с пользовательским соглашением http://www.mathnet.ru/rus/agreement

Параметры загрузки:

IP : 54.164 .48 .24

26 апреля 2023 г., 15:41:21

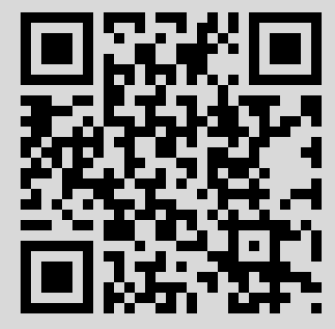




\title{
МЕТОД СУММИРОВАНИЯ РАСХОДЯЩИХСЯ РЯДОВ С ЛюБОЙ ТОЧНОСТЬЮ
}

\author{
В.В. Белокуров, Ю.П. Соловьёв, Е. Т. Шавгулидзе
}

\begin{abstract}
Предложен новый метод суммирования с любой точностью широкого класса расходящихся рядов с использованием только конечного числа членов ряда.

Библиограффия: 8 названий.
\end{abstract}

В настоящей работе рассматривается задача суммирования специального класса расходящихся рядов, которые могут быть представлены как ряды Тейлора гладких вполне монотонных функций. Под суммой ряда при этом понимается по определению значение функции в соответствующей точке.

В работе предлагается метод нахождения значения функции в этой точке с любой точностью, исходя из конечного числа членов ряда, количество которых зависит от заданной точности. Этот метод применим, в частности, для суммирования рядов с факториальным ростом членов. (Как известно, ряды такого типа возникают в задачах квантовой физики, например, при асимптотических разложениях функциональных интегралов.)

Стандартным методом суммирования таких рядов является метод Бореля и его модификации [1], [2]. Однако эти методы требуют знания всех членов ряда и не позволяют восстановить значение суммы по конечному числу членов ряда, которое обычно только и бывает известно в реальных задачах.

Предлагаемый метод суммирования расходящихся рядов представляет собой развитие метода вычисления функциональных интегралов с помощью апшроксимации их сходящимися рядами [3]-[6].

Напомним сначала определение гладкой вполне монотонной функции. Функция $f(t)$ назьвается гладкой вполне монотонной функиией на полупрямой $[0, \infty)$, если она бесконечно дифференцируема и $(-1)^{n} f^{(n)}(t) \geqslant 0$ для всех $n \geqslant 0$ и $t \in[0, \infty)$.

Нам понадобится следующая известная теорема.

Теорема (С.Н. Бернштейна). Пусть $f(t)$ - гладкая вполне монотонная функиия на полупрямой $[0, \infty)$. Тогда на полупрямой $[0, \infty)$ существует неотричательная мера Радона $\mu$ такая, что

$$
f(t)=\int_{0}^{\infty} e^{-t x} \mu(d x)
$$

Работа выполнена при финансовой поддержке Российского фонда фундаментальных исследований, грант № 97-01-00228. 
ДоКАЗАТЕЛЬСТво этой теоремы см., например, в [7].

Убедимся также в справедливости следующего предложения.

ПредЛОЖЕНИЕ 1. Пусть $f \in C^{\infty}[0, \infty)$ - вполне монотонная функиия на полупрямой $[0, \infty)$. Тогда

$$
f^{(n)}(0)=(-1)^{n} \int_{0}^{\infty} x^{n} \mu(d x) .
$$

ДокАЗАТЕЛЬСТво. Поскольку при фиксированном $t>0$ имеет место неравенство

$$
\left|x^{n} e^{-t x}\right| \leqslant n^{n} e^{-n} t^{-n},
$$

из теоремы Лебега следует, что

$$
f^{(n)}(t)=(-1)^{n} \int_{0}^{\infty} x^{n} e^{-t x} \mu(d x) .
$$

Фиксируем теперь натуральное число $n$ и рассмотрим монотонно убывающую последовательность положительных чисел $\left\{t_{k}\right\}, k \in \mathbb{N}$, для которой

$$
\lim _{k \rightarrow \infty} t_{k}=0
$$

Тогда для последовательности функций $\lambda_{k}(x)=x^{n} e^{-t_{k} x}$ при всех $x \geqslant 0$ выполняются неравенства $\lambda_{k+1}(x) \geqslant \lambda_{k}(x)$. При этом

$$
\lim _{k \rightarrow \infty} \lambda_{k}(x)=x^{n}
$$

В то же время

$$
\lim _{k \rightarrow \infty} \int_{0}^{\infty} \lambda_{k}(x) \mu(d x)=(-1)^{n} \lim _{k \rightarrow \infty} f^{(n)}\left(t_{k}\right)=(-1)^{n} f^{(n)}(0),
$$

где последнее равенство следует из непрерьвности производной $f^{(n)}(t)$.

По теореме Леви в выражении

$$
\lim _{k \rightarrow \infty} \int_{0}^{\infty} \lambda_{k}(x) \mu(d x)
$$

можно поменять порядок операций интегрирования и перехода к пределу. Следовательно,

$$
f^{(n)}(0)=(-1)^{n} \int_{0}^{\infty} \lim _{k \rightarrow \infty} \lambda_{k}(x) \mu(d x)=(-1)^{n} \int_{0}^{\infty} x^{n} \mu(d x)
$$

и последний интеграл сушествует.

В дальнейшем нам понадобятся интегралы вида

$$
B_{n}=\int_{0}^{+\infty} x^{n / 2} \mu(d x) .
$$


В частности, $f^{(n)}(0)=(-1)^{n} B_{2 n}$.

Прежде чем сформулировать основные теоремы настоящей статьи, введем, как и в работах [3]-[5], функцию

$$
\widetilde{\varphi}(\rho)=\frac{1}{2 \pi} \int_{-\infty}^{+\infty} \exp \{-i \rho r\} \exp \left\{-r^{4}\right\} d r
$$

представляющую собой преобразование Фурье экспоненты $\exp \left\{-r^{4}\right\}$. Нетрудно показать [4], что $|\widetilde{\varphi}(\rho)| \leqslant \exp \left\{-\rho^{5 / 4}\right\}$.

Положим

$$
A_{2 n}(R)=(-1)^{n} \int_{-R}^{+R} \widetilde{\varphi}(\rho) \rho^{2 n} d \rho .
$$

Tеорема 1. Пусть $f \in C^{\infty}[0, \infty)$ - вполне монотонная функиия на полупрямой $[0, \infty)$. Тогда для любого натурального $N$ и всех $t>0, R>0$ имеет место равенство

$$
f(t)=\sum_{n=0}^{N} \frac{1}{(2 n) !} t^{n / 2} A_{2 n}(R) B_{n}+\Delta(t, R, N),
$$

əde

$$
|\Delta(t, R, N)| \leqslant 2 f(0) R^{-1 / 4} \exp \left(-R^{5 / 4}\right)+\beta_{N+1} \frac{2}{(2 N+3) !} t^{(N+1) / 2} R^{2 N+3} .
$$

Здесь

$$
\beta_{N+1}= \begin{cases}\left|f^{((N+1) / 2)}(0)\right|, & \text { если } N \text { нечетно } \\ \sqrt{\left|f^{(N / 2)}(0)\right|} \sqrt{\left|f^{(N / 2+1)}(0)\right|}, & \text { если } N \text { четно. }\end{cases}
$$

ДокАЗАТЕЛЬСТво.Прежде всего преобразуем функцию $f(t)$, используя тот же прием, что и в работах [3]-[5]. А именно, представим $f(t)$ в виде

$$
f(t)=\int_{0}^{\infty} e^{-t x} \mu(d x)=\int_{0}^{+\infty}\left(\int_{-\infty}^{+\infty} \widetilde{\varphi}(\rho) \exp \left\{i t^{1 / 4} \rho x^{1 / 4}\right\} d \rho\right) \mu(d x) .
$$

Нетрудно проверить, что для интеграла (8) вьполняются условия теоремы Фубини. Поэтому

$$
f(t)=\int_{-\infty}^{+\infty} \widetilde{\varphi}(\rho)\left(\int_{0}^{+\infty} \exp \left\{i t^{1 / 4} \rho x^{1 / 4}\right\} \mu(d x)\right) d \rho .
$$

Положим

$$
J(t, R)=\int_{-R}^{+R} \widetilde{\varphi}(\rho)\left(\int_{0}^{+\infty} \exp \left\{i t^{1 / 4} \rho x^{1 / 4}\right\} \mu(d x)\right) d \rho,
$$

где $R>0$. Тогда

$$
\begin{aligned}
|f(t)-J(t, R)| & \leqslant 2 \int_{R}^{+\infty}|\widetilde{\varphi}(\rho)| d \rho \int_{0}^{+\infty} \mu(d x)=2 \int_{R}^{+\infty}|\widetilde{\varphi}(\rho)| d \rho f(0) \\
& \leqslant 2 R^{-1 / 4} \exp \left(-R^{5 / 4}\right) f(0)=\theta(R)
\end{aligned}
$$

При этом $\theta(R) \rightarrow 0$ при $R \rightarrow+\infty$. 
Заметим далее, что в силу четности функции $\widetilde{\varphi}(\rho)$

$$
J(t, R)=\int_{-R}^{+R} \tilde{\varphi}(\rho)\left(\int_{0}^{+\infty} \cos \left(t^{1 / 4} \rho x^{1 / 4}\right) \mu(d x)\right) d \rho .
$$

Воспользуемся теперь следующей аппроксимацией функции $\cos \tau$ :

$$
\cos \tau=\sum_{n=0}^{N}(-1)^{n} \frac{\tau^{2 n}}{(2 n) !}+\omega(N, \tau), \quad|\omega(N, \tau)| \leqslant \frac{\tau^{2 N+2}}{(2 N+2) !}
$$

и представим выражение для $J(t, R)$ в виде суммы двух слагаемых

$$
\begin{aligned}
J(t, R)= & \sum_{n=0}^{N}\left((-1)^{n} \frac{t^{n / 2}}{(2 n) !} \int_{-R}^{+R} \tilde{\varphi}(\rho) \rho^{2 n} d \rho \int_{0}^{+\infty} x^{n / 2} \mu(d x)\right) \\
& +\int_{-R}^{+R} \widetilde{\varphi}(\rho)\left(\int_{0}^{+\infty} \omega\left(N, t^{1 / 4} \rho x^{1 / 4}\right) \mu(d x)\right) d \rho .
\end{aligned}
$$

Такое разбиение законно, поскольку каждый из интегралов существует.

В силу формулы (13) второе слагаемое в (14) оценивается следующим образом:

$$
\begin{aligned}
& \left|\int_{-R}^{+R} \tilde{\varphi}(\rho)\left(\int_{0}^{+\infty} \omega\left(N, t^{1 / 4} \rho x^{1 / 4}\right) \mu(d x)\right) d \rho\right| \\
& \quad \leqslant \frac{1}{(2 N+2) !} t^{(N+1) / 2} \int_{-R}^{+R}|\widetilde{\varphi}(\rho)| \rho^{2 N+2} d \rho \int_{0}^{+\infty} x^{(N+1) / 2} \mu(d x) \\
& \quad \leqslant \frac{1}{(2 N+2) !} t^{(N+1) / 2} \frac{2}{(2 N+3)} R^{2 N+3} B_{N+1}
\end{aligned}
$$

Если $N+1=2 K$, то $B_{N+1}=\left|f^{(K)}(0)\right|$. Если $N+1=2 K+1$, то в силу неравенства Коши-Буняковского

$$
B_{N+1} \leqslant \sqrt{\int_{0}^{+\infty} x^{K} \mu(d x) \int_{0}^{+\infty} x^{K+1} \mu(d x)}=\sqrt{\left|f^{(K)}(0)\right|} \sqrt{\left|f^{(K+1)}(0)\right|} .
$$

Соотношения (11), (14) и (15) и доказьвают утверждение теоремы, т.е. справедливость формул $(6),(7)$.

СлеДСТВИЕ 1. Пусть $f \in C^{\infty}[0, \infty)$ - вполне монотонная функиия на полупрямой $[0, \infty)$, удовлетворяющая условиям

$$
\left|f^{(n)}(0)\right| \leqslant C n^{(4-2 \delta) n}
$$

где $C$ и $\delta$ - некоторые полохсительные постоянные. Тогда для любого $t>0 u$ любого в $>0$ найдутся $R=R(t, \varepsilon)$ u $N_{0}=N_{0}(t, \varepsilon, R)$ такие, что при всех $N>N_{0}$

$$
f(t)=\sum_{n=0}^{N} \frac{1}{(2 n) !} t^{n / 2} A_{2 n}(R) B_{n}+\Delta(t, R, N),
$$

əде $|\Delta(t, R, N)| \leqslant \varepsilon$. 
ДоКАЗАТЕЛЬСТВо следствия 1 вытекает из условия (16) и формул (6), (7).

Для практического применения предложенного метода суммирования расходящихся рядов необходимо еще указать алгоритм вычисления входящих в формулу (6) коэффициентов $B_{n}$.

Для четных $n B_{n}=(-1)^{n / 2} f^{(n / 2)}(0)$. Для приближенного вычисления коэффициентов с нечетными индексами $n$ можно, например, использовать прием, примененньй в работе [6].

В настоящей работе мы опишем другой метод их вычисления. Основу его составляет

ТЕорема 2. Пусть $f \in C^{\infty}[0, \infty)$ - вполне монотонная функиия на полупрямой $[0, \infty)$. Предположимм, что

$$
\left|f^{(n)}(0)\right| \leqslant K^{n}(2 n) !
$$

где $K$ - некоторое число, большее единиць. Тогда для любых вешественных $\lambda>0 u \varepsilon>0$, найдутся натуральное число $N=N(K, \lambda, \varepsilon)$ и коэффициенты $b_{m}=b_{m}(K, \lambda, \varepsilon)$ такие, что

$$
\int_{0}^{+\infty} x^{\lambda / 2} \mu(d x)=\sum_{m=0}^{N} b_{m} f^{(m)}(0)+\delta
$$

əдe $|\delta| \leqslant \varepsilon$.

Доказательству теоремы предпошлем следующую лемму.

\section{ЛЕмма 1. Имеем}

$$
\begin{aligned}
\int_{0}^{+\infty} & x^{n+(\alpha-1) / 2} \mu(d x) \\
\quad= & \frac{1}{C(\alpha, n)} \int_{0}^{\infty}\left(\int_{0}^{\infty}\left(\cos \left(\xi x^{1 / 2}\right)-\sum_{k=0}^{n-1} \frac{(-1)^{k}}{(2 k) !} \xi^{2 k} x^{k}\right) \mu(d x)\right) \xi^{-(2 n+\alpha)} d \xi
\end{aligned}
$$

$2 \partial e$

$$
C(\alpha, n)=\frac{(-1)^{n} \pi}{2 \cos (\pi \alpha / 2) \Gamma(2 n+\alpha)}, \quad|\alpha|<1 .
$$

ДокАЗАТЕЛЬСТво. Рассмотрим интеграл

$$
\int_{0}^{\infty}\left(\int_{0}^{\infty}\left(\cos \left(\xi x^{1 / 2}\right)-\sum_{k=0}^{n-1} \frac{(-1)^{k}}{(2 k) !} \xi^{2 k} x^{k}\right) \mu(d x)\right) \xi^{-(2 n+\alpha)} d \xi .
$$

В силу теоремы Фубини в нем можно менять порядок интегрирований. Сделаем замену переменных $x^{1 / 2} \xi=y$. В результате получим, что рассматриваемый интеграл равен

$$
\int_{0}^{\infty}\left(\cos y-\sum_{k=0}^{n-1} \frac{(-1)^{k}}{(2 k) !} y^{2 k}\right) y^{-(2 n+\alpha)} d y \int_{0}^{\infty} x^{n+(\alpha-1) / 2} \mu(d x) .
$$

Для доказательства леммы теперь достаточно воспользоваться известным соотношениeM

$$
\int_{0}^{\infty}\left(\cos y-\sum_{k=0}^{n-1} \frac{(-1)^{k}}{(2 k) !} y^{2 k}\right) y^{-(2 n+\alpha)} d y=\frac{(-1)^{n} \pi}{2 \cos (\pi \alpha / 2) \Gamma(2 n+\alpha)}
$$

справедливьп при $|\alpha|<1$. 
ДОКАЗАТЕЛЬСТВО ТЕОРЕМЫ 2 . Если $\lambda=2 n$, где $n$ - натуральное число, то

$$
\int_{0}^{+\infty} x^{\lambda / 2} \mu(d x)=\int_{0}^{+\infty} x^{n} \mu(d x)=(-1)^{n} f^{(n)}(0) .
$$

Следовательно, в этом случае $N=n$, все коэффициенты $b_{m}=0$, за исключением $b_{n}=1$, и $\delta=0$.

Во всех остальных случаях $\lambda$ можно представить в виде $\lambda=2 n+\alpha-1$, где $n-$ натуральное число и $|\alpha|<1$.

Пусть $a_{1}$ и $a_{2}$ - некоторые положительные числа, удовлетворяющие неравенствам

$$
\begin{gathered}
a_{1}<a_{2}, \quad 0<a_{1}<\frac{1}{\sqrt{K}} \\
a_{2}>\left(\frac{2}{\varepsilon|C(\alpha, n)|(2 n+\alpha-1)}\right)^{1 /(2 n+\alpha-1)} .
\end{gathered}
$$

Представим интеграл в правой части формулы (20) в виде суммы трех интегралов

$$
\begin{aligned}
\int_{0}^{+\infty} & x^{n+(\alpha-1) / 2} \mu(d x) \\
= & \frac{1}{C(\alpha, n)}\left(\int_{0}^{a_{1}}\left(\int_{0}^{\infty}\left(\cos \left(\xi x^{1 / 2}\right)-\sum_{k=0}^{n-1} \frac{(-1)^{k}}{(2 k) !} \xi^{2 k} x^{k}\right) \mu(d x)\right) \xi^{-(2 n+\alpha)} d \xi\right. \\
& +\int_{a_{1}}^{a_{2}}\left(\int_{0}^{\infty}\left(\cos \left(\xi x^{1 / 2}\right)-\sum_{k=0}^{n-1} \frac{(-1)^{k}}{(2 k) !} \xi^{2 k} x^{k}\right) \mu(d x)\right) \xi^{-(2 n+\alpha)} d \xi \\
& \left.+\int_{a_{2}}^{\infty}\left(\int_{0}^{\infty}\left(\cos \left(\xi x^{1 / 2}\right)-\sum_{k=0}^{n-1} \frac{(-1)^{k}}{(2 k) !} \xi^{2 k} x^{k}\right) \mu(d x)\right) \xi^{-(2 n+\alpha)} d \xi\right)
\end{aligned}
$$

Из выбора пределов интегрирования следует, что

$$
\begin{aligned}
& \frac{1}{C(\alpha, n)} \int_{a_{2}}^{\infty}\left(\int_{0}^{\infty}\left(\cos \left(\xi x^{1 / 2}\right)-\sum_{m=0}^{n-1} \frac{(-1)^{m}}{(2 m) !} \xi^{2 m} x^{m}\right) \mu(d x)\right) \xi^{-(2 n+\alpha)} d \xi \\
& =\frac{1}{C(\alpha, n)} \sum_{m=0}^{n-1} \frac{(-1)^{m}}{(2 m) !} \frac{a_{2}^{2 m-2 n-\alpha+1}}{(2 m-2 n-\alpha+1)} \int_{0}^{\infty} x^{m} \mu(d x) \\
& \quad+\frac{1}{C(\alpha, n)} \int_{a_{2}}^{\infty}\left(\int_{0}^{\infty} \cos \left(\xi x^{1 / 2}\right) \mu(d x)\right) \xi^{-(2 n+\alpha)} d \xi \\
& =\frac{1}{C(\alpha, n)} \sum_{m=0}^{n-1} \frac{1}{(2 m) !} \frac{a_{2}^{2 m-2 n-\alpha+1}}{(2 m-2 n-\alpha+1)} f^{(m)}(0)+\delta_{1},
\end{aligned}
$$

где

$$
\delta_{1}=\frac{1}{C(\alpha, n)} \int_{a_{2}}^{\infty}\left(\int_{0}^{\infty} \cos \left(\xi x^{1 / 2}\right) \mu(d x)\right) \xi^{-(2 n+\alpha)} d \xi
$$


В силу неравенства (24)

$$
\left|\delta_{1}\right| \leqslant \frac{1}{|C(\alpha, n)|} \int_{a_{2}}^{\infty} \xi^{-(2 n+\alpha)} d \xi<\frac{\varepsilon}{2} .
$$

Рассмотрим теперь интеграл

$$
\frac{1}{C(\alpha, n)} \int_{0}^{a_{1}}\left(\int_{0}^{\infty}\left(\cos \left(\xi x^{1 / 2}\right)-\sum_{k=0}^{n-1} \frac{(-1)^{k}}{(2 k) !} \xi^{2 k} x^{k}\right) \mu(d x)\right) \xi^{-(2 n+\alpha)} d \xi
$$

Пользуясь разложением (13), этот интеграл можно представить в виде

$$
\begin{aligned}
& \frac{1}{C(\alpha, n)} \int_{0}^{a_{1}}\left(\int_{0}^{\infty}\left(\sum_{m=n}^{N_{1}-1} \frac{(-1)^{m}}{(2 m) !} \xi^{2 m} x^{m}+\omega\left(N_{1}, \xi x^{1 / 2}\right)\right) \mu(d x)\right) \xi^{-(2 n+\alpha)} d \xi \\
& =\frac{1}{C(\alpha, n)} \sum_{m=n}^{N_{1}-1} \frac{1}{(2 m) !} \frac{a_{1}^{2 m-2 n-\alpha+1}}{(2 m-2 n-\alpha+1)} f^{(m)}(0)+\delta_{2},
\end{aligned}
$$

где

$$
\delta_{2}=\frac{1}{C(\alpha, n)} \int_{0}^{a_{1}}\left(\int_{0}^{\infty} \omega\left(N_{1}, \xi x^{1 / 2}\right) \mu(d x)\right) \xi^{-(2 n+\alpha)} d \xi
$$

а натуральное число $N_{1}$ выбирается из условия

$$
\left|\frac{1}{C(\alpha, n)} \frac{1}{\left(2 N_{1}\right) !} \frac{a_{1}^{2 N_{1}-2 n-\alpha+1}}{\left(2 N_{1}-2 n-\alpha+1\right)} f^{\left(N_{1}\right)}(0)\right|<\frac{\varepsilon}{4} .
$$

В этом случае

$$
\begin{aligned}
\left|\delta_{2}\right| & \leqslant \frac{1}{|C(\alpha, n)|} \frac{1}{\left(2 N_{1}\right) !} \int_{0}^{a_{1}}\left(\int_{0}^{\infty} x^{2 N_{1}} \mu(d x)\right) \xi^{2 N_{1}-2 n-\alpha} d \xi \\
& =\left|\frac{1}{C(\alpha, n)} \frac{1}{\left(2 N_{1}\right) !} \frac{a_{1}^{2 N_{1}-2 n-\alpha+1}}{\left(2 N_{1}-2 n-\alpha+1\right)} f^{\left(N_{1}\right)}(0)\right|<\frac{\varepsilon}{4} .
\end{aligned}
$$

Займемся теперь интегралом

$$
\frac{1}{C(\alpha, n)} \int_{a_{1}}^{a_{2}}\left(\int_{0}^{\infty}\left(\cos \left(\xi x^{1 / 2}\right)-\sum_{k=0}^{n-1} \frac{(-1)^{k}}{(2 k) !} \xi^{2 k} x^{k}\right) \mu(d x)\right) \xi^{-(2 n+\alpha)} d \xi
$$

Существование для него представления вида (19) вытекает из следующих двух лемм и равенства

$$
\cos t=2 \cos ^{2} \frac{t}{2}-1
$$

которое позволяет представить функцию $\cos t$ в виде полинома $P\left(\cos ^{2}\left(2^{-l} t\right)\right)$ для произвольного натурального числа $l$. 
ЛЕМма 2. Пусть

$$
I_{s}=\int_{a_{1}}^{a_{2}}\left(\int_{0}^{\infty}\left(\cos \left(2^{-l} \xi x^{1 / 2}\right)\right)^{2 s} P\left(\xi^{2} x\right) \mu(d x)\right) d \xi,
$$

әде $P\left(\xi^{2} x\right)$ - полином от $\xi^{2} x$ с вещественными коэффициентами, предель интегрирования $a_{1}$ и а а 2 удовлетворяют соответственно неравенствам (23) $u(24), s$ и l-неотрицательные иелье числа.

Если l таково, что

$$
l>1+\log _{2}\left(a_{2} K\right),
$$

то для любого $\varepsilon_{1}>0$ найдется $M$ такое, что имеет место следующее представление:

$$
I_{s}=\sum_{r=0}^{M} \frac{(-1)^{r}}{(2 r) !} \int_{a_{1}}^{a_{2}}\left(\int_{0}^{\infty}\left(\cos \left(2^{-l} \xi x^{1 / 2}\right)\right)^{2 s-2} P\left(\xi^{2} x\right) \xi^{2 r} x^{r} 2^{-2 r(l-1)-1} \mu(d x)\right) d \xi+\sigma
$$

əде $|\sigma|<\varepsilon_{1}$.

ДокАЗАТЕЛЬСтво. Легко видеть, что для доказательства леммы достаточно соответствуюшее утверждение доказать лишь для подынтегральных выражений вида

$$
\left(\cos \left(2^{-l} \xi x^{1 / 2}\right)\right)^{2 s} \xi^{2 k} x^{k}
$$

где $k$ - неотрицательное целое число.

Имеем

$$
\begin{aligned}
\left(\cos \left(2^{-l} \xi x^{1 / 2}\right)\right)^{2 s} \xi^{2 k} x^{k}= & \left(\cos \left(2^{-l} \xi x^{1 / 2}\right)\right)^{2 s-2}\left(\cos \left(2^{-l} \xi x^{1 / 2}\right)\right)^{2} \xi^{2 k} x^{k} \\
= & \left(\cos \left(2^{-l} \xi x^{1 / 2}\right)\right)^{2 s-2}\left(\sum_{r=0}^{M} \frac{(-1)^{r}}{(2 r) !} \xi^{2 r+2 k} x^{r+k} 2^{-2 r(l-1)-1}\right. \\
& \left.+\frac{(-1)^{M+1}}{(2(M+1)) !} \xi^{2(M+1)+2 k} x^{(M+1)+k} 2^{-2(M+1)(l-1)-1} \cos \theta\right),
\end{aligned}
$$

где $0<\theta<2^{-l} \xi x^{1 / 2}$.

Получим теперь оценку для остатка $\sigma$ :

$$
\begin{aligned}
|\sigma| & \leqslant \frac{1}{(2 M+2) !} \int_{a_{1}}^{a_{2}}\left(\int_{0}^{\infty} \xi^{2(M+1)+2 k} x^{(M+1)+k} 2^{-2(M+1)(l-1)-1} \mu(d x)\right) d \xi \\
& \leqslant \frac{\left|f^{(M+k+1)}(0)\right| a_{2}^{2 M+2 k+3}}{(2 M+2) !(2 M+2 k+3) 2^{2(M+1)(l-1)+1}} .
\end{aligned}
$$

В силу неравенств (18) и (25) имеем

$$
\begin{aligned}
|\sigma| & \leqslant \frac{K^{M+k+1}(2 M+2 k+2) ! a_{2}^{2 M+2 k+3}}{(2 M+2) !(2 M+2 k+3) 2^{2(M+1)(l-1)+1}} \\
& \leqslant \frac{K^{M+k+1}(2 M+2 k+2) ! a_{2}^{2 M+2 k+3}}{2(2 M+2) !(2 M+2 k+3) K^{2 M+2} a_{2}^{2 M+2}} \\
& =\frac{(2 M+3) \cdots(2 M+2 k+2) a_{2}^{2 k+1}}{2(2 M+2 k+3) K^{M-k+1}} .
\end{aligned}
$$

Так как $K>1$, из этого неравенства следует, что для достаточно большого $M$ абсолютная величина $\sigma$ может быть сделана сколь угодно малой. Тем самым, лемма 2 доказана. 
Лемма 3. В предположсениях леммы 2 для любого $\varepsilon_{2}>0$ существуют натуральное число $J$ и коэффииченты $b_{j}, j=0, \ldots, J$, такие, что

$$
I_{s}=\sum_{j=0}^{J} b_{j} f^{(j)}(0)+\gamma
$$

əде $|\gamma|<\varepsilon_{2}$.

ДоКАЗАТЕЛЬСТВО леммы проводится индукцией по $s$.

Для $s=0$ утверждение леммы очевидно. В самом деле,

$$
\begin{aligned}
I_{0} & =\int_{a_{1}}^{a_{2}}\left(\int_{0}^{\infty} P\left(\xi^{2} x\right) \mu(d x)\right) d \xi \\
& =\int_{a_{1}}^{a_{2}}\left(\int_{0}^{\infty}\left(p_{0}+p_{1} \xi^{2} x+p_{2}\left(\xi^{2} x\right)^{2}+\cdots+p_{m}\left(\xi^{2} x\right)^{m}\right) \mu(d x)\right) d \xi \\
& =\sum_{j=0}^{m} p_{j} \int_{a_{1}}^{a_{2}} \xi^{2 j} d \xi \int_{0}^{\infty} x^{j} \mu(d x)=\sum_{j=0}^{m} p_{j} \frac{a_{2}^{2 j+1}-a_{1}^{2 j+1}}{2 j+1} f^{(j)}(0) .
\end{aligned}
$$

Предполагая теперь утверждение вьполненным для $s$, докажем его для $s+1$. Для этого воспользуемся леммой 2 , положив в ней $\varepsilon_{1}=\varepsilon_{2} / 2$. Имеем

$$
\begin{aligned}
I_{s+1} & =\sum_{r=0}^{M} \frac{(-1)^{r}}{(2 r) !} \int_{a_{1}}^{a_{2}}\left(\int_{0}^{\infty}\left(\cos \left(2^{-l} \xi x^{1 / 2}\right)\right)^{2 s} P\left(\xi^{2} x\right) \xi^{2 r} x^{r} 2^{-2 r(l-1)-1} \mu(d x)\right) d \xi+\sigma \\
& =\int_{a_{1}}^{a_{2}}\left(\int_{0}^{\infty}\left(\cos \left(2^{-l} \xi x^{1 / 2}\right)\right)^{2 s} Q\left(\xi^{2} x\right) \xi^{2 r} x^{r} 2^{-2 r(l-1)-1} \mu(d x)\right) d \xi+\sigma,
\end{aligned}
$$

где

$$
Q\left(\xi^{2} x\right)=\sum_{r=0}^{M} \frac{(-1)^{r}}{(2 r) !} P\left(\xi^{2} x\right) \xi^{2 r} x^{r} 2^{-2 r(l-1)-1}
$$

и $|\sigma|<\varepsilon_{1}=\varepsilon_{2} / 2$.

По предположению индукции существуют натуральное число $J$ и коэффициенты $b_{j}$, $j=0, \ldots, J$, такие, что

$$
\int_{a_{1}}^{a_{2}}\left(\int_{0}^{\infty}\left(\cos \left(2^{-l} \xi x^{1 / 2}\right)\right)^{2 s} Q\left(\xi^{2} x\right) \xi^{2 r} x^{r} 2^{-2 r(l-1)-1} \mu(d x)\right) d \xi=\sum_{j=0}^{J} b_{j} f^{(j)}(0)+\widetilde{\gamma}
$$

где $|\widetilde{\gamma}|<\varepsilon_{1}$. Следовательно,

$$
I_{s+1}=\sum_{j=0}^{J} b_{j} f^{(j)}(0)+\widetilde{\gamma}+\sigma
$$

причем $|\widetilde{\gamma}+\sigma| \leqslant|\widetilde{\gamma}|+|\sigma|<\varepsilon_{1}+\varepsilon_{1}=\varepsilon_{2}$.

Это завершает доказательство леммы 3 и вместе с ней теоремы 2 .

Заметим, что в доказательстве теоремы 2 по существу содержится алгоритм для вычисления величин $N=N(K, \lambda, \varepsilon)$ и $b_{m}=b_{m}(K, \lambda, \varepsilon), 1 \leqslant m \leqslant N$, в зависимости от значений параметров $K, \lambda, \varepsilon$. 
СлЕДСТВИЕ 2. При произвольных значениях параметров $K>0, \varepsilon>0 u 0 \leqslant t \leqslant T$ найдутся натуральное число $M=M(K, \varepsilon, T)$ и набор коэффичиентов $c_{m}=c_{m}(K$, $\varepsilon, t), 1 \leqslant m \leqslant M$, такие, что для любой функиии $f(t)$, удовлетворяюшей условиям теоремы 2, выполняется неравенство

$$
\left|f(t)-\sum_{m=0}^{M} c_{m} f^{(m)}(0)\right|<\varepsilon .
$$

ДоказАТЕЛьСтво. Для данных $K>0, \nu>0, T>0$ выберем наименьшее натуральное число $L=L(K, \nu, T)$, удовлетворяющее неравенствам

$$
L \geqslant 2 \sqrt{K T}\left(\ln \frac{2}{\nu}\right)^{8 / 5}, \quad L \geqslant \frac{1}{\ln 2}\left(\ln \frac{1}{\nu}+\frac{2}{5} \ln \ln \frac{2}{\nu}\right)
$$

и положим $R(\nu)=(\ln (2 / \nu))^{4 / 5}$.

В силу следствия 1 получаем, что

$$
\begin{aligned}
& \left|f(t)-\sum_{n=0}^{J(K, \nu, T)} \frac{1}{(2 n) !} t^{n / 2} A_{2 n}(R(\nu)) B_{n}\right| \\
& \leqslant R^{-1 / 4} \exp \left(-R^{5 / 4}\right)+(K T)^{(N+1) / 2} \frac{(N+2) !}{(2 N+3) !} R^{2 N+3} \\
& \leqslant \frac{\nu}{2}+\left(\frac{\sqrt{K T}(\ln (2 / \nu))^{8 / 5}}{N+1}\right)^{N+1}\left(\ln \frac{2}{\nu}\right)^{2 / 5} \leqslant \nu .
\end{aligned}
$$

Возьмем теперь $\nu=\varepsilon / 2$ и для всех $k$, удовлетворяющих неравенствам

$$
1 \leqslant 2 k+1 \leqslant L\left(K, \frac{\varepsilon}{2}, T\right)
$$

определим числа $\nu_{k}$ :

$$
\nu_{k}=\frac{\varepsilon(2(2 k+1)) ! T^{-(2 k+1) / 2}}{2 L(K, \varepsilon / 2, T)\left(\left|A_{2(2 k+1)}(R(\varepsilon / 2))\right|+1\right)} .
$$

Из теоремы 2 следует, что

$$
\begin{aligned}
& \left|\frac{t^{(2 k+1) / 2} A_{2(2 k+1)}(R(\varepsilon / 2))}{(2(2 k+1)) !}\left(B_{2 k+1}-\sum_{m=0}^{N\left(K,(2 k+1) / 2, \nu_{k}\right)} b_{m}\left(K, \frac{2 k+1}{2}, \nu_{k}\right) f^{(m)}(0)\right)\right| \\
& \quad \leqslant \frac{\varepsilon}{2 L(K, \varepsilon / 2, T)}
\end{aligned}
$$

Видно, что в качестве $M(K, \varepsilon, T)$ можно выбрать

$$
M=\max \left\{L\left(K, \frac{\varepsilon}{2}, T\right) ; N\left(K, \frac{2 k+1}{2}, \nu_{k}\right)\right\} .
$$


При этом коэффициенты $c_{m}=c_{m}(K, \varepsilon, t), 1 \leqslant m \leqslant M$, равны

$$
\begin{aligned}
c_{m}= & \frac{1}{(4 m) !} t^{m} A_{2 m}\left(R\left(\frac{\varepsilon}{2}\right)\right) \\
& +\sum_{k=0}^{L(K, \varepsilon / 2, T)-1} b_{m}\left(K, \frac{2 k+1}{2}, \nu_{k}\right) \frac{1}{(2(2 k+1)) !} t^{(2 k+1) / 2} A_{2(2 k+1)}\left(R\left(\frac{\varepsilon}{2}\right)\right) .
\end{aligned}
$$

Теорема 2 позволяет восстановить значение функции $f(t)$ с любой точностью, используя конечное число членов ряда, и дает тем самьг эффективньй алгоритм суммирования широкого класса расходящихся рядов.

Укажем теперь, какие ряды могут быть отнесены к этому классу. Рассмотрим степенной ряд

$$
\sum_{n=0}^{\infty} \frac{(-1)^{n}}{n !} u_{n} t^{n}
$$

коэффициенты которого образуют две позитивные последовательности

$$
\left\{u_{0}, u_{1}, \ldots\right\}, \quad\left\{u_{1}, u_{2}, \ldots\right\}
$$

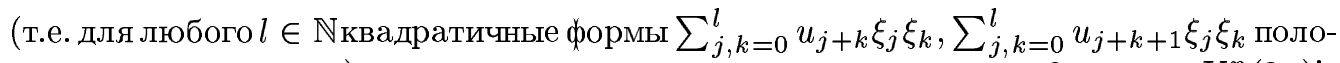
жительно определены) и удовлетворяют следующим неравенствам: $0 \leqslant u_{n} \leqslant K^{n}(2 n)$ ! .

В этом случае ряд (28) является рядом Тейлора некоторой гладкой вполне монотонной функции $f(t)\left(u_{n}=(-1)^{n} f^{(n)}(0)\right)$ [8], причем эта функция единственна [1].

Отметим, что при указанных условиях значение функции $f(t)$ совпадает с суммой ряда в смысле Бореля [1]. Принципиальное же отличие предлагаемого нами метода от метода Бореля состоит в следующем. Для суммирования методом Бореля необходимо найти аналитическое продолжение функции

$$
u(x)=\sum_{n=0}^{\infty} \frac{(-1)^{n}}{(n !)^{2}} u_{n} t^{n} x^{n}
$$

построенной с использованием всех коэффициентов ряда. Чаще всего это проделать не удается, и, более того, в реальных задачах, как правило, известно только конечное число коэффициентов $u_{n}$. Предложенньй в настоящей работе метод позволяет для любого значения $t$ с любой точностью найти сумму ряда (28), зная лишш конечное число членов ряда.

В качестве примера приведем результаты приближенного суммирования рядов

$$
S_{\alpha}=\sum_{n=0}^{\infty}(-1)^{n}(n !)^{\alpha}, \quad 0 \leqslant \alpha \leqslant 1 .
$$

При $\alpha=0$ и $\alpha=1$ - это знаменитые расходящиеся ряды

$$
S_{0}=\sum_{n=0}^{\infty}(-1)^{n}, \quad S_{1}=\sum_{n=0}^{\infty}(-1)^{n} n ! .
$$


Традиционные методы суммирования [1] дают следующие значения:

$$
S_{0}=\frac{1}{2}, \quad S_{1}=0.596 \ldots
$$

Ряды для других $\alpha$ традиционными методами просуммировать не удается.

Предложенньй в работе метод позволяет просуммировать с любой точностью эти ряды для произвольных $\alpha$. Приведем значения некоторых из $S_{\alpha}$, вычисленные с точностью 0.001 (для получения указанной точности в формуле (17) выбиралось $R=11$, $N=40, t=1)$ :

$$
\begin{gathered}
S_{0}=0.500 \ldots, \quad S_{0,1}=0.511 \ldots, \quad S_{0,2}=0.521 \ldots, \quad S_{0,3}=0.532 \ldots, \\
S_{0,4}=0.542 \ldots, \quad S_{0,5}=0.552 \ldots, \quad S_{0,6}=0,561 \ldots, \quad S_{0,7}=0.570 \ldots, \\
S_{0,8}=0.579 \ldots, \quad S_{0,9}=0.588 \ldots, \quad S_{1}=0,596 \ldots
\end{gathered}
$$

\section{СПИСОК ЦИТИРОВАННОЙ ЛИТЕРАТУРЫ}

[1] Харди Г. Расходящиеся ряды. М.: ИЛ, 1951.

[2] Dingle R. B. Asymptotic Expansions. London-New York: Acad. Press, 1973.

[3] Belokurov V.V., Shavgulidze E.T., Solovyov Yu. P. New perturbation theory for quantum field theory: convergent series instead of asymptotic expansions // Mod. Phys. Lett. A. 1995. V. 10. № 39. P. 3033-3041.

[4] Белокуров В. В., Соловьёв Ю. П., Шавгулидзе Е. Т. Вычисление функциональных интегралов с помощью сходящихся рядов // Фундамент. и прикл. матем. 1997. Т. 3.№3. C. $693-713$.

[5] Белокуров В. В., Соловьёв Ю. П., Шавгулидзе Е. Т. Теория возмущений со сходящимися рядами для функциональных интегралов по фейнмановской мере // УМН. 1997. Т. 52. №2. C. 153-154.

[6] Belokurov V. V., Kamchatny V. V., Shavgulidze E. T., Solovyov Yu. P. Perturbation theory with convergent series for arbitrary values of coupling constant // Mod. Phys. Lett. A. 1997. V. 12. № 10. P. 661-672.

[7] Эдвардс Р. Функциональный анализ. М.: Мир, 1969.

[8] Крейн М. Г., Нудельман А. А. Проблема моментов Маркова и экстремальные задачи. М.: Наука, 1973.

Московский государственный университет им. М.В. Ломоносова

Поступило

E-mail: belokur@rector.msu.su, solo@difgeo.math.msu.su, 11.09 .1998

shav@mech .math.msu.su

Исправленный вариант

13.01 .2000 